\title{
Using Multiple Contrast Mechanisms via Forescatter Electron Channeling Contrast Imaging to Resolve the Burgers Vector of GaN Threading Dislocations
}

\author{
Y.N. Picard,* M. E. Twigg, ${ }^{*}$ J. D. Caldwell, ${ }^{*}$ C. R. Eddy, Jr., * M.A. Mastro, ${ }^{*}$ and R.T. Holm* \\ *Electronics Science and Technology Division, Naval Research Laboratory, Washington, DC 20375
}

Electron channeling contrast imaging (ECCI) is a scanning electron microscopy (SEM) technique capable of imaging individual dislocations in crystalline materials. Similar to transmission electron microscopy (TEM), ECCI employs diffraction contrast to image extended defects. ECCI holds many promising advantages as a rapid, non-destructive alternative for dislocation analysis. A readily accessible approach to ECCI utilizes forescatter diode detectors mounted on commercially available electron backscatter diffraction (EBSD) systems [1-2]. Additional advantages to using this forescatter approach includes enhanced topographic contrast sufficient to image atomic steps [3] as well as orientation contrast sensitive to texture within crystalline materials.

In this study, forescatter ECCI is employed to investigate threading dislocations in heteroepitaxial $\mathrm{GaN}$ thin film surfaces. Specimens are tilted so that the $20 \mathrm{keV}$ incident electron beam is $\sim 25^{\circ}$ incident to the sample surface. Also, the specimens are oriented so that the acting diffraction condition is $\mathrm{g}=\underline{2} 11 \underline{\mathrm{6}}$. Forescatter ECCI images show that atomic steps can be readily imaged (Fig.1). In addition, individual channeling contrast features across the film surface are observed in the form of characteristic intensity fluctuations. Two distinct types of intensity fluctuations can be differentiated based on the relative strength of the observed contrast. The stronger intensity fluctuations are often centered at the beginning of an atomic step or atomic step spiral (Fig. 2), a phenomenon strongly tied to screw dislocation (SD) behavior. Directionality of the intensity fluctuations is found to consistently correlate with the directionality of clockwise and counterclockwise atomic step spirals. Rotational direction of atomic step spirals are a function of the Burgers vector: $b= \pm c[0001]$. Hence, directionality of the observed intensity fluctuations can be directly tied to the specific SD Burgers vector.

When the sample is tilted to within $0.1^{\circ}$ of the $g=\underline{2} 11 \underline{6}$ Bragg angle, orientation contrast can be observed across the textured GaN film surface (Fig. 3). Shifts in the grayscale across the surface designate the location of low-angle grain boundaries (GBs). Most of the weaker intensity fluctuations can be found decorating these low-angle GBs, a strong indication that the weaker intensity fluctuations are edge dislocations. The observed directionality of these weaker intensity fluctuations show a correspondence to the GB and indicate a direct relationship to the suspected edge dislocation's Burgers vector. Simulations based on solutions to the Howie-Whelan equations qualitatively verify these experimental observations. The observed ECCI diffraction contrast for SDs are found to be consistent with previous plan-view TEM studies.

\section{References}

[1] C. Trager-Cowan et al., Phys. Rev. B 75 (2007) 085301.

[2] Y. N. Picard et al., Appl. Phys. Lett. 90 (2007). 234101.

[3] Y. N. Picard and M. E. Twigg, J. Appl. Phys., 104 (2008) 124906. 


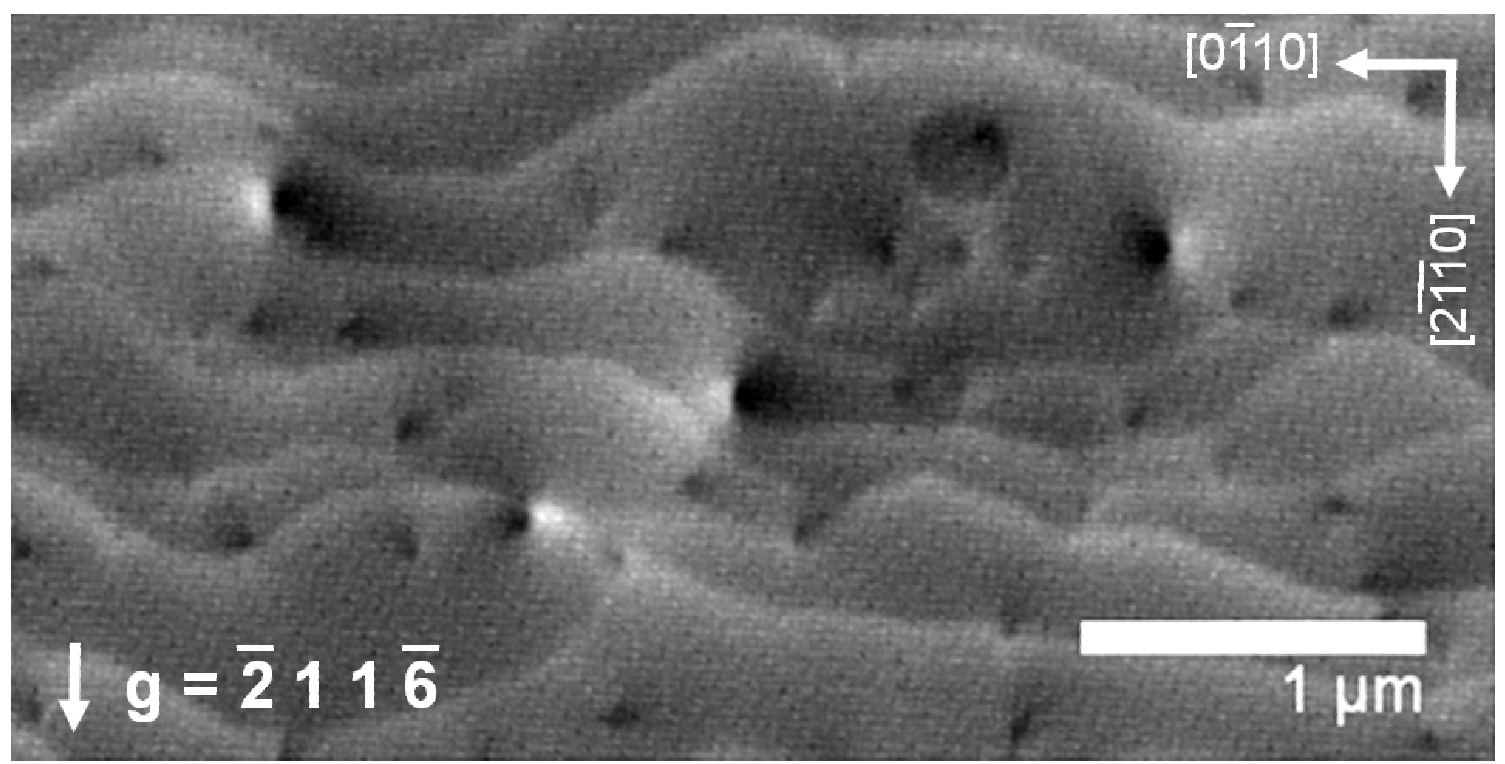

Fig. 1. ECCI micrograph of GaN surface showing both strong and weak intensity fluctuations.
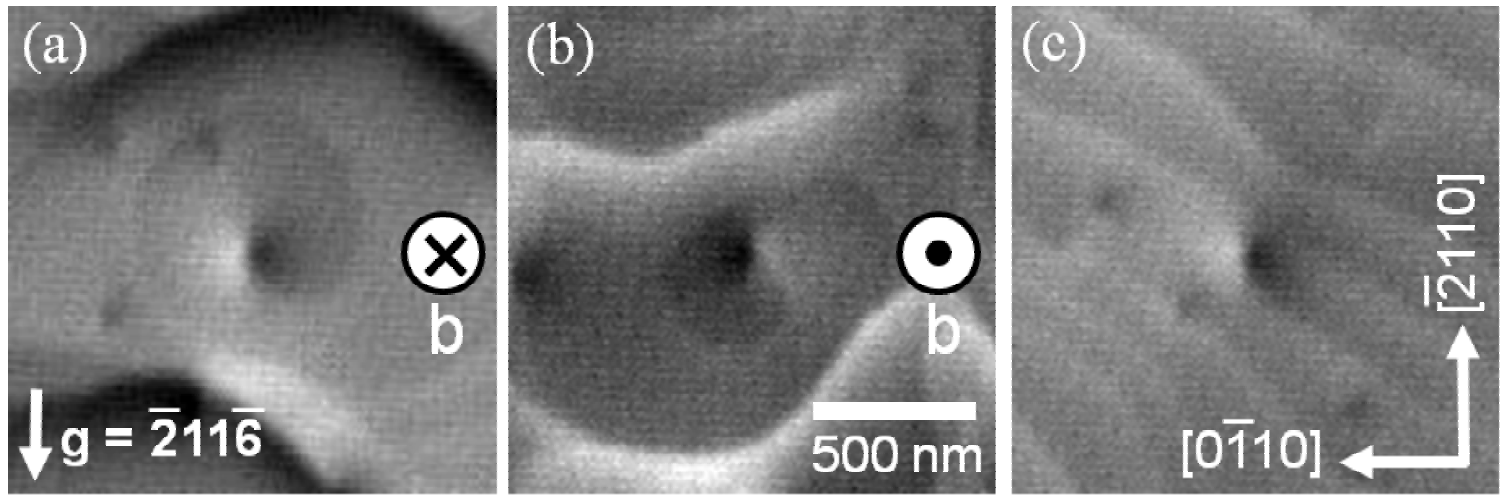

Fig. 2. ECCI micrographs centered on suspected SDs. Based on the (a) counter-clockwise and (b) clockwise rotation of atomic step spirals, the Burgers vector direction can be assigned.

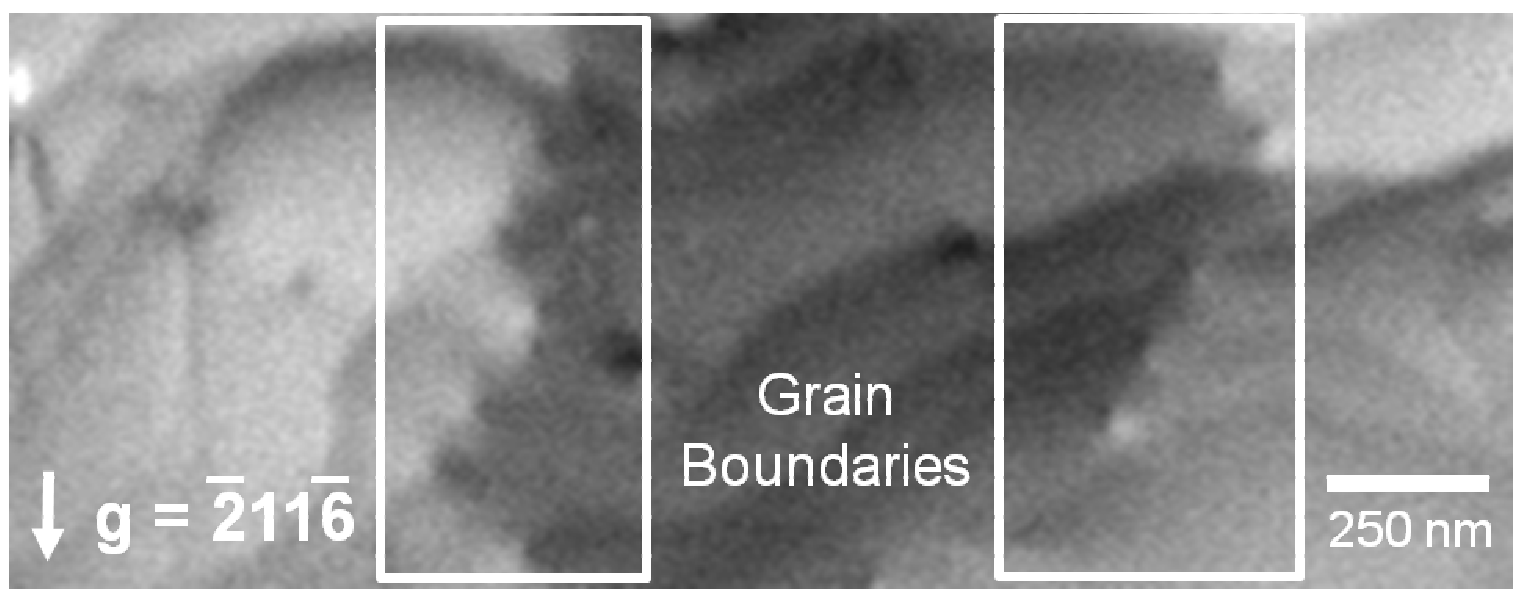

Fig. 3. ECCI micrograph of GaN surface exhibiting orientation contrast. Lines of transition from one gray scale to another indicate the location of low-angle grain boundaries. Weaker intensity fluctuations are observed along these low-angle grain boundaries and are suspected to denote edge dislocations. 